\title{
Performance and comparison of fiber vibration sensing using SAC-OCDMA with direct decoding techniques
}

\begin{abstract}
Intensity modulated fiber vibrations sensing using Khazani Syed (KS) and Modified Quadratic Congruence Code (MQC) code for quasi distributed vibration measurement have been evaluated. The systems employed Spectral Direct (SD) Decoding for Spectral Amplitude Coding-Optical Code Division Multiple Access (SAC-OCDMA). SAC-OCDMA is adopted because of its enormous impact in dealing with Multiple Access Interference (MAI). The setup was carried out for three sensor points in three consecutive measurements. The performance of the sensing system with the KS and MQC codes were compared at the same weight and number of code sequence. It was observed that the applied vibration frequencies were obtained at the receivers and the signal employing MQC are received at slightly better power than KS code. However, KS code setup employed less number of components and therefore, reduce the cost and complexity of achieving quasi-distributed vibration sensing.
\end{abstract}

Keyword: Khazani Syed (KS) Code; Spectral Direct (SD); Spectral Amplitude CodingOptical Code Division Multiple Access (SAC-OCDMA); Multiple Access Interference (MAI); Vibration sensing 